\title{
Determination of the uncertainty of mass flow measurement using the orifice for different values of the Reynolds number
}

\author{
Anna Golijanek-Jędrzejczyk ${ }^{1, *}$, Andrzej Mrowiec $^{2}$, Robert Hanus ${ }^{3}$, Marcin Zych $^{4}$, and Dariusz Świsulski ${ }^{1}$ \\ ${ }^{1}$ Gdańsk University of Technology, Faculty of Electrical and Control Engineering, 80-233 Gdańsk, Poland \\ ${ }^{2}$ The President Stanisław Wojciechowski State University of Applied Sciences in Kalisz, Polytechnic Faculty, 62-800 Kalisz, \\ Poland \\ ${ }^{3}$ Rzeszów University of Technology, Faculty of Electrical and Computer Engineering, 35-959 Rzeszów, Poland \\ ${ }^{4}$ AGH University of Science and Technology, Faculty of Geology, Geophysics and Environmental Protection, 30-059 Kraków, \\ Poland
}

\begin{abstract}
Standard orifice flowmeters are widely used in the chemical and energy industry. Therefore, it is essential to know how accurate the measurements made with these instruments are. The paper presents an estimation of measurement uncertainty of a liquid mass flow using the orifice plate. The authors will present the influence of ranges of the Reynolds number on the estimated uncertainty, obtained on the basis of simulation and laboratory experiments. The research was conducted for the central orifice in the Reynolds number $8,000<\operatorname{Re}<21,000$. The results of estimating the extended uncertainty of the measurement of water flow using simulation and experimental method, are convergent. The maximum difference in the extended uncertainty values of flow measurement for the simulation and experiment was $0.04 \cdot 10^{-3} \mathrm{~kg} / \mathrm{s}$.
\end{abstract}

\section{Introduction}

Despite the wide development of flow meters, including non-standard flow meters (e.g. slotted, perforated holes [1]), standard orifices are still often chosen and applied in measuring solutions.

The venturi method using a measuring orifice is one of the most popular methods for measuring both mass flow of fluids and gases. It is estimated that this is the most commonly used method in industrial measurements (over $40 \%$ of the market [2]), in industries such as: oil and gas,the chemical and nuclear and energy industries. For example, in the Chinese oil and gas industry, this type of flowmeters accounts for approximately $95 \%$ of all flow meters used [2].

The advantages of orifices in relation to other solutions are: simple construction, high reliability, ruggedness, the possibility of being used in wide ranges of pressure and temperature and low cost [2-6].

Therefore, it is important to know how precisely these devices can measure and what factors affect the accuracy of this measurement.

This article presents the results of uncertainty estimation of water flow measurement obtained by Monte Carlo simulation. These results were compared with the results obtained from experimental tests carried out on the hydrodynamic installation, in which a standard (centric) orifice was installed.

The aim of the study was to determine the influence of the Reynolds number in the range of $8,000<\mathrm{Re}$ $<21,000$ on the estimated uncertainty of flow measurement.

\section{The study}

The orifice is a thin disc mounted in a pipe so that the axis of the bore coincides with the axis of the tube (Fig. 1).

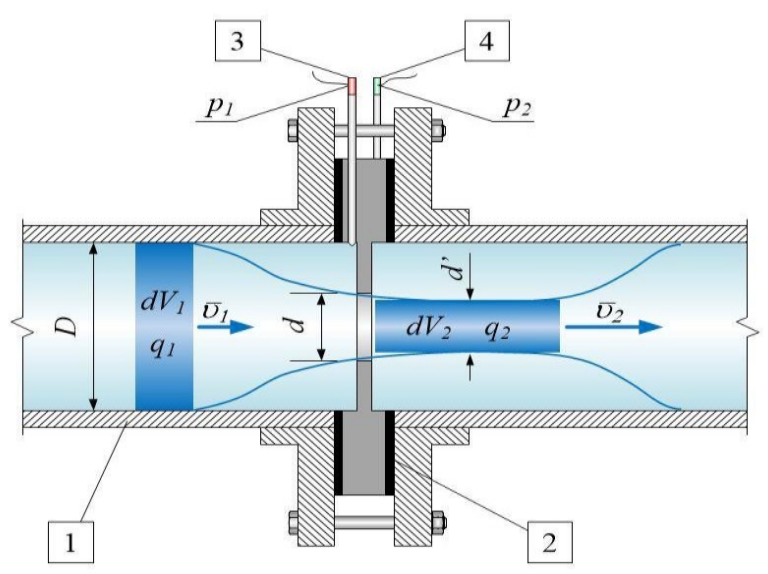

Fig. 1. Fluid flow through a measurement orifice: 1 - pipe, $2-$ orifice, 3, 4-pressure gauges.

The relation between the volume flow $q$ and the pressure difference $\Delta p$ can be determined by the transformation of potential energy (pressure) into kinetic energy (velocity) at a flow through the reducer. During this process, it is assumed that the continuity of the fluid stream is constant and its density does not change during the passage through different cross sections.

\footnotetext{
${ }^{*}$ Corresponding author: anna.golijanek-jedrzejczyk@pg.edu.pl
} 
The conditions allowing the determination of the mass flow rate $q$ are as follows [7]:

$$
q=\frac{c}{\sqrt{1-\left(\frac{d}{D}\right)^{4}}} \varepsilon \cdot \frac{\pi}{4} \cdot d^{2} \cdot \sqrt{2 \cdot \Delta p \cdot \rho_{1}}
$$

where:

$C$ - a discharge coefficient [-],

$\varepsilon$ - compressibility of different fluids [-],

$\Delta p$ - the differential pressure [Pa],

$\rho_{1}$ - density of medium $\left[\mathrm{kg} / \mathrm{m}^{3}\right]$,

$\beta$-relation between the orifice and the pipe diameter:

$$
\beta=d / D \quad[-],
$$

$d$ - orifice diameter [m],

$D$ - pipe diameter [m].

The analysis of the uncertainty of measurement of the mass flow $q$ was carried out under the following assumptions:

- at any point in any cross section perpendicular to the axis of the flow, a continuous stream of the fluid flow is maintained and equal to the velocity,

- incompressible fluid without internal friction and a constant density $\rho_{1}=\rho_{2}=$ const.,

- the medium is a fluid, that is a ratio $\varepsilon=1$,

- minimum of 30 measurements were made on the basis of which the $n=30$ observations of flow stream $q$ were achieved.

The aim of the research was to determine the estimate of the measured flow $q$ (arithmetic mean value) and the corresponding expanded uncertainty $U(q)$ for the coverage factor $k_{p}=2.00$ (which corresponds to approximately $95 \%$ probability of expansion), according to the formula:

$$
U(q)=k_{p} \cdot u_{c}(q)
$$

for different streams.

The assessment of the expanded uncertainty $U(q)$ requires the first estimation of complex uncertainty $u_{\mathrm{c}}(q)$. Assuming no correlation between the measured quantities, according to the law of the uncertainty propagation, complex uncertainty $u_{\mathrm{c}}(q)$ determining the mass flow $q$ is defined as follows [7]:

$$
\begin{gathered}
u_{c}(q)=\sqrt{u_{A}^{2}(q)+u_{B}^{2}(q)}= \\
=\sqrt{u_{A}^{2}(q)+\left(\frac{\partial q}{\partial C}\right)^{2} \cdot u^{2}(C)+\left(\frac{\partial q}{\partial d}\right)^{2} \cdot u^{2}(d)+} \\
+\left(\frac{\partial q}{\partial D}\right)^{2} \cdot u^{2}(D)+\left(\frac{\partial q}{\partial \Delta p}\right)^{2} \cdot u^{2}(\Delta p)+ \\
+\left(\frac{\partial q}{\partial \rho_{1}}\right)^{2} \cdot u^{2}\left(\rho_{1}\right)
\end{gathered}
$$

where:

$u_{A}(q)$ - the estimated uncertainty of measurement of the mass flow $q$ with the Type A method,

$u_{B}(q)$ - the estimated uncertainty of measurement of the mass flow $q$ with the Type B method,

$u(C)$ - the uncertainty in the discharge coefficient $C$, $u(d)$ - the uncertainty of orifice diameter measurement,
$u(D)$ - the uncertainty of pipe diameter measurement, $u(\Delta p)$ - the uncertainty of the differential pressure measurement,

$u\left(\rho_{1}\right)$ - the uncertainty of the density of medium measurement.

To determine Type A uncertainty, the normal distribution is assumed (because the number of measurements is greater than 30 ).

The standard uncertainty $u_{A}(q)$ can be evaluated as:

$$
u_{A}(q)=\sqrt{\frac{1}{n \cdot(n-1)} \cdot \sum_{i=1}^{n}\left(q_{i}-\bar{q}\right)^{2}}
$$

The weight coefficients appearing in the $u_{B}(q)$ (3) (partial derivatives) have been determined and are presented in [7].

The estimation of uncertainty $u(C), u(d), u(D), u(\Delta p)$ and $u\left(\rho_{1}\right)$ requires knowledge of the relative errors $\delta$ with which these parameters are determined. It is also necessary to accept the probability distributions of these parameters.

The following probability distributions for the input quantities: for discharge coefficient $C$ - the normal distribution, while for the other parameters: $d, D, \Delta p, \rho_{1}$ - the rectangular distributions were assumed.

\subsection{Tested orfice}

Experimental research was conducted with a centric orifice with an inner diameter $\mathrm{d}$ of $25.005 \mathrm{~mm}$.

Measurement $d$ was made using the Wenzel LH 65 coordinate measuring machine with an uncertainty of $2.5 \mu \mathrm{m}$.

Simulation and experimental tests were carried out for the data presented in Table 1.

Table 1. Data.

\begin{tabular}{|c|c|c|c|}
\hline & Parameter & Unit & Value \\
\hline 1 & $d$ & {$[\mathrm{~m}]$} & 0.025005 \\
\hline 2 & $D$ & {$[\mathrm{~m}]$} & 0.050000 \\
\hline 3 & $\varepsilon$ & {$[-]$} & 1.000000 \\
\hline 4 & $\beta$ & {$[-]$} & 0.500100 \\
\hline
\end{tabular}

The research was carried out for the orifice in the range of Reynolds numbers $R e=8,000 \ldots 21,000$, for temperature in range $T=(295.55 \ldots 301.35) \mathrm{K}$.

\section{Simulation results}

The article first presents the results of simulation studies of the mass flow uncertainty estimation using the Monte Carlo numerical method (MC). 
This method is used to verify the estimates of analytical uncertainty $[7,8]$ especially in cases of indirect measurements and when the measuring function is non-linear.

For this purpose, the random number generator from Microsoft Excel was used. It is assumed that the function of measuring mass flow, according to equation (5) is:

$$
\begin{aligned}
q= & \bar{q}+c_{0} \cdot u_{A}(q)+c_{1} \cdot u(C)+c_{2} \cdot u(d)+ \\
& +c_{3} \cdot u(D)+c_{4} \cdot u(\Delta p)+c_{5} \cdot u\left(\rho_{1}\right)
\end{aligned}
$$

where $c_{1}$ to $c_{5}$ mark sensitivity coefficients, and the coefficient $c_{0}$ is equal to 1 . The rest of the coefficients are defined in [7].

The assumed probability distributions and the relative errors $\delta$ of the various parameters measurements are presented in Table 2. The errors $\delta$ are the maximum relative permission errors of used devices for each parameter.

Table 2. The values of the relative errors $\delta$.

\begin{tabular}{|c|c|c|c|}
\hline & Variance & Error $\delta$ [\%] & Distribution \\
\hline 1 & $u^{2}(C)[-]$ & 0.50 & normal \\
\hline 2 & $u^{2}(d)\left\lfloor\mathrm{m}^{2}\right\rfloor$ & 0.01 & \\
\cline { 1 - 2 } 3 & $u^{2}(D)\left\lfloor\mathrm{m}^{2}\right\rfloor$ & 0.02 & \multirow{2}{*}{ rectangular } \\
\cline { 1 - 2 } 4 & $u^{2}(\Delta p)\left\lfloor P a^{2}\right\rfloor$ & 0.15 & \\
\cline { 1 - 2 } 5 & $u^{2}\left(\rho_{1}\right)\left[\frac{\mathrm{kg}^{2}}{\mathrm{~m}^{6}}\right]$ & 3.55 & \\
\hline
\end{tabular}

For the above-mentioned data, estimations were performed in Microsoft Excel of uncertainty using the Monte Carlo method for 8 different flow streams $q$. The number of samples $M$ was equal to $10^{4}$.

Figure 2 presents the values of the arithmetic mean of flow $q$ in the function of the Reynolds number.

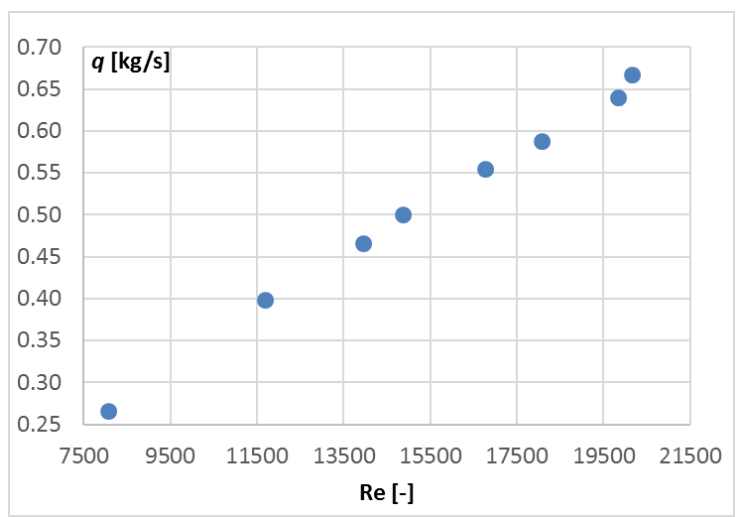

Fig. 2. Flow values $q$ as a function of the Reynolds number.

Figure 3 shows the example of probability density function and histogram of the simulated numerical values for the mass flow $q=0.5004 \mathrm{~kg} / \mathrm{s}$. a)

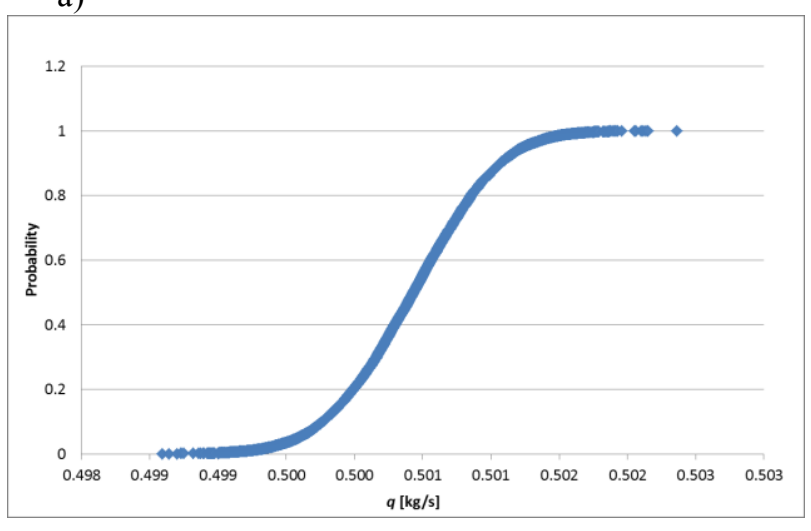

b)

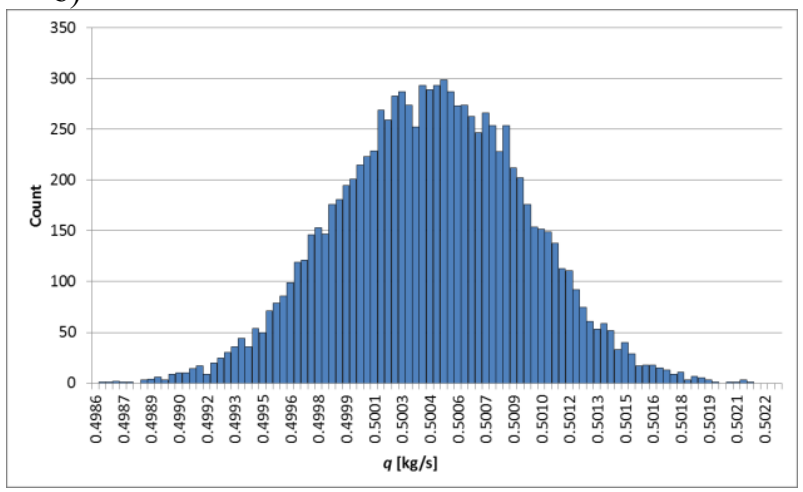

Fig. 3. Simulated outflow distribution (a) and histogram (b) for the mass flow $q=0.5004 \mathrm{~kg} / \mathrm{s}$.

The results of estimating the expanded uncertainties $U(q)$ for all measuring points together with the flow estimate $q$ are summarized in Table 3 in the following convention: $(\bar{q} \pm U(q))\lfloor\mathrm{kg} / \mathrm{s}\rfloor$.

Table 3. The results of mass flow estimation from Monte Carlo simulation.

\begin{tabular}{|c|c|}
\hline No & $\begin{array}{c}\text { Mass flow estimation } \boldsymbol{q} \\
10^{-3}[\mathrm{~kg} / \mathrm{s}]\end{array}$ \\
\hline 1 & $(265.93 \pm 0.88)$ \\
\hline 2 & $(398.58 \pm 0.64)$ \\
\hline 3 & $(466.44 \pm 0.84)$ \\
\hline 4 & $(500.40 \pm 0.93)$ \\
\hline 5 & $(554.56 \pm 0.98)$ \\
\hline 6 & $(587.66 \pm 1.01)$ \\
\hline 7 & $(639.87 \pm 1.13)$ \\
\hline 8 & $(666.84 \pm 1.16)$ \\
\hline
\end{tabular}

The simulation results obtained using the Monte Carlo method showed that in the investigated range of Reynolds numbers, the expanded uncertainty $U(q)$ of the flow measurement is of the order of $1 \cdot 10^{-3} \mathrm{~kg} / \mathrm{s}$. The smallest uncertainty $U(q)=0.64 \cdot 10^{-3} \mathrm{~kg} / \mathrm{s}$ was obtained for $q=398.58 \cdot 10^{-3} \mathrm{~kg} / \mathrm{s}$, and the largest $U(q)=1.17 \cdot 10^{-}$ ${ }^{3} \mathrm{~kg} / \mathrm{s}$ for $q=666.84 \cdot 10^{-3} \mathrm{~kg} / \mathrm{s}$. 


\section{Experimental results}

Experimental tests of the orifices were carried out on a laboratory stand, the diagram of which is shown in Fig. 4.

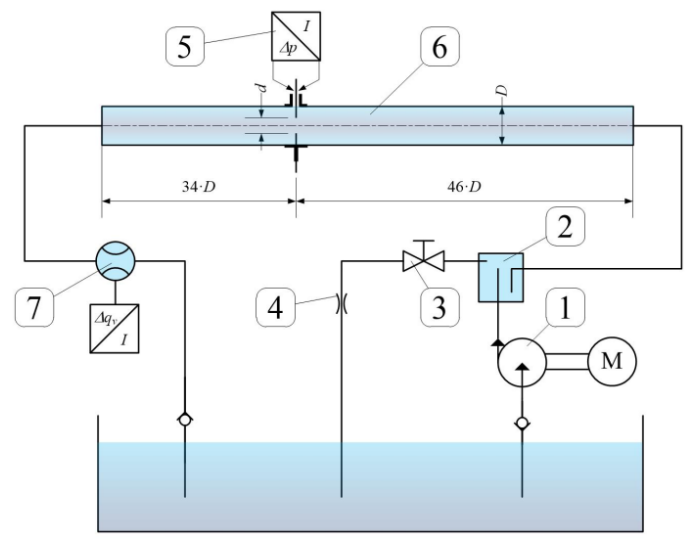

Fig. 4. Scheme of the laboratory stand (description in the text).

The water flow in the presented installation was forced by a centrifugal pump (1) in a closed system. A stream of water flowed through the vent (2), wherein followed by separation of air bubbles. They were discharged along with a part of the stream through the valve (3) and a stepped lateral vent (exchangeable bleed (4)), while regulating the stream of flowing water in the measuring system. The main part of the water stream was flowing through a measuring pipe (6) (stainless steel with an inner diameter of $D=50 \mathrm{~mm}$ ), in which a standard orifice with a coefficient $\beta=0.5$ was installed. Dynamic pressure on the orifice was measured with the differential pressure transmitter APR 2000/ ALW (5) programmed with a measuring range of $\Delta p=2.4 \mathrm{kPa}$, at which the relative error is $0.15 \%$ of the base.

Experimental studies were carried out for the same data as in the case of simulations.

Figure 5 shows the expanded uncertainties $U(q)$ of the mass flow both obtained by simulation (MC) and from the measurements.

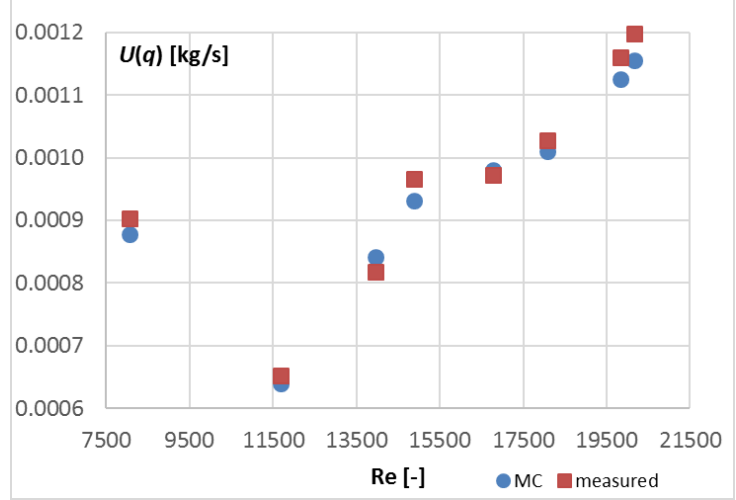

Fig. 5. Comparison of the extended uncertainty $U(q)$ for the simulation and experiment.

Figure 5 shows that in the studied range of Reynolds numbers $R e=8,000 \ldots 21,000$, the expanded uncertainty
$U(q)$ values for simulation and experiment are convergent. The maximum difference in flow uncertainty values for simulations and experiments was $0.04 \cdot 10^{-3} \mathrm{~kg} / \mathrm{s}$ for $q=666.85 \cdot 10^{-3} \mathrm{~kg} / \mathrm{s}$.

\section{Summary}

The article presents the problem of estimation of uncertainty of a liquid mass flow measurement using the centric orifice plate for different values of the Reynolds number.

The authors carried out the estimation of the uncertainty of the extended flow measurement $q$ simulationally - using the Monte Carlo numerical method, and then conducted a series of experiments on the laboratory stand for the same input data. The achieved results from both studies are very similar.

In the studied range of Reynolds numbers $8,000<\mathrm{Re}$ $<21,000$ the expanded uncertainty $U(q)$ is at the level of $1 \cdot 10^{-3} \mathrm{~kg} / \mathrm{s}$. The smallest value of $U(q)$ was obtained for $q=398.58 \cdot 10^{-3} \quad \mathrm{~kg} / \mathrm{s} \quad$ (simulation: $0.64 \cdot 10^{-3} \mathrm{~kg} / \mathrm{s}$, experiment: $\left.0.65 \cdot 10^{-3} \mathrm{~kg} / \mathrm{s}\right)$, the largest for $q=666.85 \cdot 10^{-3} \mathrm{~kg} / \mathrm{s} \quad$ (simulation: $\quad 1.17 \cdot 10^{-3} \mathrm{~kg} / \mathrm{s}$, experiment: $\left.1.2010^{-3} \mathrm{~kg} / \mathrm{s}\right)$. The maximum difference in flow uncertainty values for simulations and experiments was $0.04 \cdot 10^{-3} \mathrm{~kg} / \mathrm{s}$ for $q=666.85 \cdot 10^{-3} \mathrm{~kg} / \mathrm{s}$.

The research confirmed that the influence of the Reynolds number on the determination of uncertainty in the mass flow flux measurement is significant. With the increase of the Reynolds number, the uncertainty of the flow measurement increases.

The convergence of simulation and experimental results confirm the usefulness of the Monte Carlo simulation in estimating the uncertainty of the flow measurement using the orifice.

\section{References}

1. V.K. Singh, T. John Tharakan, Flow Measurement and Instrumentation 45, 375-383 (2015)

2. J. Dong, C. Jing, Y. Peng, Y. Liu, H. Ren, and X. Liu, Flow Measurement and Instrumentation 59, 52-62 (2018)

3. F. Shan, Z. Liu, W. Liu, Y. Tsuji, Chemical Engineering Science 152, 497-506 (2016)

4. D. Kasprzak, A. Mrowiec, Pomiary Automatyka Robotyka 20, 25-28 (2016)

5. E. Wróblewska, P. Synowiec, W. Wędrychowicz, A. Andruszkiewicz, Przegląd Elektrotechniczny 94(8), 154-157 (2018)

6. M. Straka, A. Fiebach, T. Eichler, C. Koglin, Flow Measurement and Instrumentation 60, 124-133 (2018)

7. A. Golijanek-Jędrzejczyk, D. Świsulski, R. Hanus, M. Zych, L. Petryka, Flow Measurement and Instrumentation 62, 84-92 (2018)

8. A. Dzwonkowski, A. Golijanek-Jędrzejczyk, L. Rafiński, Przeglad Elektrotechniczny 91(8), 11-14 (2015) 